\title{
Effects of proton beam irradiation on mitochondrial biogenesis in a human colorectal adenocarcinoma cell line
}

\author{
BYUNG GEUN HA, SUNG SUK JUNG and YUN HEE SHON
}

Bio-Medical Research Institute, Kyungpook National University Hospital, Daegu 700-721, Republic of Korea

Received January 15, 2017; Accepted June 27, 2017

DOI: 10.3892/ijo.2017.4067

\begin{abstract}
Proton beam therapy has recently been used to improve local control of tumor growth and reduce side-effects by decreasing the global dose to normal tissue. However, the regulatory mechanisms underlying the physiological role of proton beam radiation are not well understood, and many studies are still being conducted regarding these mechanisms. To determine the effects of proton beams on mitochondrial biogenesis, we investigated: mitochondrial DNA (mtDNA) mass; the gene expression of mitochondrial transcription factors, functional regulators, and dynamic-related regulators; and the phosphorylation of the signaling molecules that participate in mitochondrial biogenesis. Both the mtDNA/nuclear DNA (nDNA) ratio and the mitochondria staining assays showed that proton beam irradiation increases mitochondrial biogenesis in 12-O-tetradecanoylphorbol-13-acetate (TPA)-induced aggressive HT-29 cells. Simultaneously, proton beam irradiation increases the gene expression of the mitochondrial transcription factors PGC-1 $\alpha$, NRF1, ERR $\alpha$, and mtTFA, the dynamic regulators DRP1, OPA1, TIMM44, and TOM40, and the functional regulators CytC, ATP5B and CPT1- $\alpha$. Furthermore, proton beam irradiation increases the phosphorylation of AMPK, an important molecule involved in mitochondrial biogenesis that is an energy sensor and is regulated by the AMP/ATP ratio. Based on these findings, we suggest that proton beam irradiation inhibits metastatic potential by increasing mitochondrial biogenesis and function in TPA-induced aggressive HT-29 cells.
\end{abstract}

Correspondence to: Professor Yun Hee Shon, Bio-Medical Research Institute, Kyungpook National University Hospital, 44-2 Samduk 2ga Jung-gu, Daegu 700-721, Republic of Korea E-mail: yhshon@hmail.knu.ac.kr

Abbreviations: TPA, 12-O-tetradecanoylphorbol-13-acetate; PGC-1 $\alpha$, peroxisome proliferator-activated receptor co-activator $1 \alpha$; NRF, nuclear respiratory factor; mtTFA, mitochondrial transcription factor A; ERR $\alpha$, estrogen receptor-related receptor alpha; AMPK, AMP-activated protein kinase; PBT, proton beam therapy

Key words: colorectal cancer, proton radiotherapy, mitochondrial biogenesis, peroxisome proliferator-activated receptor co-activator 1 alpha, AMP-activated protein kinase, metastasis

\section{Introduction}

Colorectal cancer (CRC) is steadily increasing worldwide, but has a particularly high incidence in developed countries. Approxiamtely $20 \%$ of CRC patients already have metastases at diagnosis (1). It is well known that metastasis spreads cancer to other organs such as the liver, lungs and lymph nodes, and it accounts for over $90 \%$ of cancer-related mortality (2).

Mitochondria play an important role in the production of ATP via oxidative phosphorylation (OXPHOS). Mitochondria are also involved in biosynthetic metabolic processes involving cholesterol, heme, lipids and nucleotides. Moreover, mitochondria are considered the main sources of reactive oxygen species and nitrogen species (3). Mitochondrial biogenesis is a complex process that occurs by the cooperation of numerous genes between nuclear and mitochondrial genomes. It is regulated by a complex transcriptional network of key factors including peroxisome proliferator-activated receptor co-activator 1 alpha (PGC-1 $\alpha$ ), the nuclear respiratory factors (NRFs), estrogen receptor-related receptor alpha (ERR $\alpha)$ and mitochondrial transcription factor A (mtTFA) (4).

Research has shown an association between mitochondrial biogenesis and metastasis. Cells with depleted mitochondrial DNA (mtDNA) exhibit increased invasiveness through the upregulation of cathepsin L expression (5). Depletion of mtDNA also promotes invasion by modulating the expression of matrix metalloproteinases, tissue inhibitors of the MMP family and other invasion-related genes (6). However, increased mitochondrial biogenesis is induced by treatment with bezafibrate, a pan agonist of peroxisome proliferator-activated receptors (PPARs), in cancer cells such as 143B, HeLa and MDA-MB-231. Bezafibrate treatment reduces cell growth, glucose utilization and invasion in these cells (7). When wildtype KISS1, a metastasis suppressor, is upregulated, aerobic glycolysis decreases and OXPHOS predominates. KISS1expressing cells show that increased mitochondrial biogenesis is accompanied by increased mitochondrial gene expression and higher expression of PGC-1 $\alpha$, a key co-activator that regulates mitochondrial mass and metabolism (8).

Proton beam therapy (PBT), an alternative to gamma or X-ray irradiation therapy, has produced promising clinical results worldwide (9). In particular, PBT has recently been used to treat hepatocellular carcinoma (10), non-small cell lung (11), prostate (12) and head and neck tumors (13). Some in vitro studies have shown that PBT inhibits metastatic characteris- 
tics such as the increase of adhesion and migration $(14,15)$. In previous studies, we also found that proton beam irradiation inhibits metastatic potentials such as migration, invasion and the expression of MMP-2 and MMP-9 in HT-29 cells $(16,17)$. Furthermore, proton beam irradiation inhibits gene expression and the activities of molecules involved in integrin trafficking and integrin-mediated signaling, which are essential processes in tumor progression (18). However, the contribution of abnormal mitochondrial biogenesis to colon cancer metastasis is unknown. In the present study, we investigated the effects of proton beam irradiation on mitochondrial biogenesis by determining: mtDNA mass; the expression of mitochondriaspecific transcription factors, dynamic regulators, and functional molecules; and the activities of signaling molecules in 12-O-tetradecanoyl phorbol-13-acetate (TPA)-induced aggressive HT-29 cells.

\section{Materials and methods}

Materials. The following items were purchased from the stated commercial sources: TPA (P1585) and 4',6-diamidino2-phenylindole (DAPI, D9542) were from Sigma-Aldrich Co. (St. Louis, MO, USA); rabbit anti-human phospho AMPK (\#2535), mouse anti-human AMPK (\#2793), and rabbit anti-human phospho SIRT1 (\#2314) antibodies were from Cell Signaling Technology (Danvers, MA, USA); rabbit anti-human SIRT1 (sc-15404), rabbit anti-human PGC-1 $\alpha$ (sc-13067), mouse anti-human $\beta$-actin (sc-47778), goat antimouse IgG-horseradish peroxidase (HRP) (sc-2005), and goat anti-rabbit IgG-HRP (sc-2004) secondary antibodies were from Santa Cruz Biotechnology Inc., (Santa Cruz, CA, USA); ECL Plus Western Blotting Substrate (34580) was from Pierce Biotechnology (Rockford, IL, USA); TRIzol (15596026) and MitoTracker Red CMXRos (M7512) were from Invitrogen-Life Technologies (Carlsbad, CA, USA); the PrimeScript First Strand cDNA Synthesis kit (\#6110A) was from Takara Bio Inc. (Shiga, Japan); the FastStart Universal SYBR-Green Master Mix (04 913850 001) was from Roche Applied Science (Mannheim, Germany); and the Phosphatase Inhibitor Cocktail (P3200) and Protease Inhibitor Cocktail (P3100) solutions were from GenDEPOT (Barker, TX, USA).

Cell culture. As previously described in detail (18), the HT-29 cell line was purchased from the Korean Cell Line Bank (KCLB no. 30038, Seoul, Republic of Korea). Cells were maintained in $5 \% \mathrm{CO}_{2}$ at $37^{\circ} \mathrm{C}$ in Roswell Park Memorial Institute (RPMI-1640) medium including $10 \%$ fetal bovine serum (FBS; SH30084.03; HyClone Laboratories-GE Healthcare Life Science, Victoria, Australia) and 100 U/ml penicillin-streptomycin (15140122; Invitrogen). For induction of metastatic capacity, cells were incubated with $150 \mathrm{nM}$ TPA for $1 \mathrm{~h}$ and then RPMI-1640 medium was added before proton beam irradiation.

Proton beam irradiation. According to previously described methods (19), cell irradiation was performed with a $100-\mathrm{MeV}$ proton beam using the $100 \mathrm{MeV}$ linac equipment (Scanditronix, Uppsala, Sweden) at the Korea Multi-purpose Accelerator Complex (KOMAC, Gyeongju, Republic of Korea). Briefly, cells in a $12.5-\mathrm{cm}^{2}$ flask filled with RPMI-1640 medium were placed on a beam stage and irradiated (1, 4 and $8 \mathrm{~Gy})$ at the center of the Bragg peaks modulated to $6-\mathrm{cm}$ widths. The dose during each beam irradiation was measured using radiochromic film (GAF-MD55; GAF Corp., Wayne, NJ, USA) as an in situ measuring tool.

Mitochondrial biogenesis analysis. We cultured the irradiated cells for 48 and $72 \mathrm{~h}$ to investigate the effect of proton beam irradiation on mitochondrial biogenesis. As previously described in detail (20), DNA from cells was extracted using a genomic DNA purification kit (A1120; Promega Corp., Madison, WI, USA), according to the manufacturer's instructions. Mitochondrial biogenesis was determined by the ratio of cytochrome $c$ oxidase subunit I (COX I) for mtDNA to ribosomal protein large p0 (RPLP0) for nuclear DNA (nDNA), quantified by quantitative reverse transcription polymerase chain reaction (RT-qPCR). Data were normalized against the expression of RPLP0.

Mitochondrial staining and quantification. As previously described in detail (20), the irradiated cells were cultured in 8-well chamber slides (12-565-8; Thermo Fisher Scientific, Rochester, NY, USA) for 48 and $72 \mathrm{~h}$. After washing with phosphate-buffered saline (PBS), the cells were incubated with RPMI-1640 medium containing $20 \mathrm{nM}$ MitoTracker Red CMXRos at $37^{\circ} \mathrm{C}$ for $30 \mathrm{~min}$. After washing with PBS, the cells were fixed with $4 \%$ paraformaldehyde for $10 \mathrm{~min}$, and stained with $1 \mu \mathrm{g} / \mathrm{ml}$ DAPI for $10 \mathrm{~min}$. Fluorescent images were obtained using a digital fluorescence microscope (Nikon Eclipse 80i; Nikon, Tokyo, Japan) at x400 magnification. For quantification of the fluorescent signal, the cells were dissolved with a lysis buffer $(0.1 \mathrm{M}$ potassium phosphate, $1 \%$ Triton X-100, $\mathrm{pH} 10.0$ ) for $10 \mathrm{~min}$. Dimethyl sulfoxide (DMSO) was added to the lysates, which were then incubated for $10 \mathrm{~min}$. The fluorescent signal intensity from the lysates was measured using a microplate reader (FLUOstar OPTIMA; BMG Labtech, Ortenberg, Germany) at $466 \mathrm{~nm}$ excitation and $540 \mathrm{~nm}$ emission.

$R T$ - $q$ PCR analysis. As previously described in detail (20), total RNA from cells was obtained using TRIzol (Invitrogen), and cDNA was produced using a PrimeScript First strand cDNA Synthesis kit (Takara Bio Inc.) according to the manufacturer's instructions. To analyze gene expression, RT-qPCR was conducted using a FastStart SYBR-Green Master mix (Roche Diagnostics) in an ABI Prism 7300 Sequence Detection System (Applied Biosystems, Foster City, CA, USA). Levels of target gene expression were calculated relative to the expression level of the endogenous reference gene, $\beta$-actin, using the delta cycle threshold method (Table I).

Western blot analysis. As previously described in detail (20), the irradiated cells were cultured for 48 and $72 \mathrm{~h}$, and lysed in radio-immunoprecipitation assay (RIPA) buffer (89900; Thermo Fisher Scientific, Waltham, MA, USA). Total protein lysates were separated by $8 \%$ SDS-polyacrylamide gel electrophoresis and transferred to nitrocellulose membranes (10 401 396; Whatman GmbH, Dassel, Germany). The membranes were incubated with $5 \%$ non-fat milk for $1 \mathrm{~h}$ at $25^{\circ} \mathrm{C}$, and incubated with the primary antibody $(1: 1,000)$ 
A
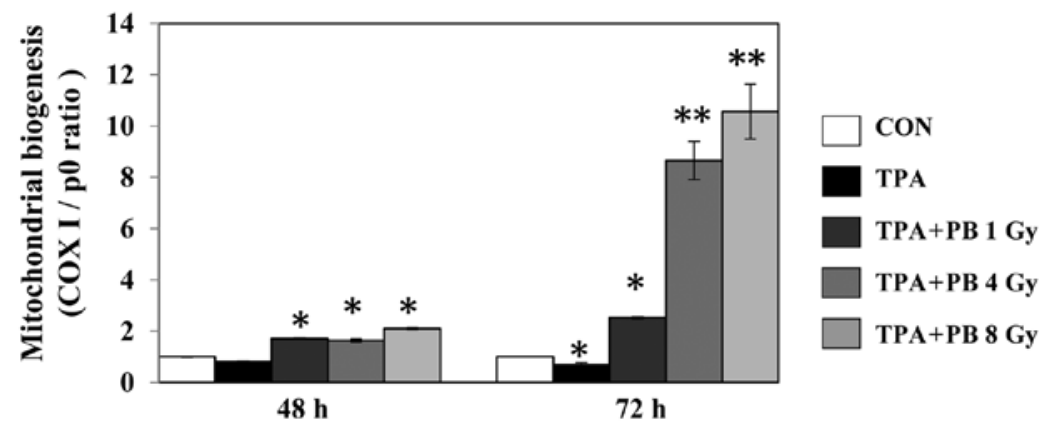

B
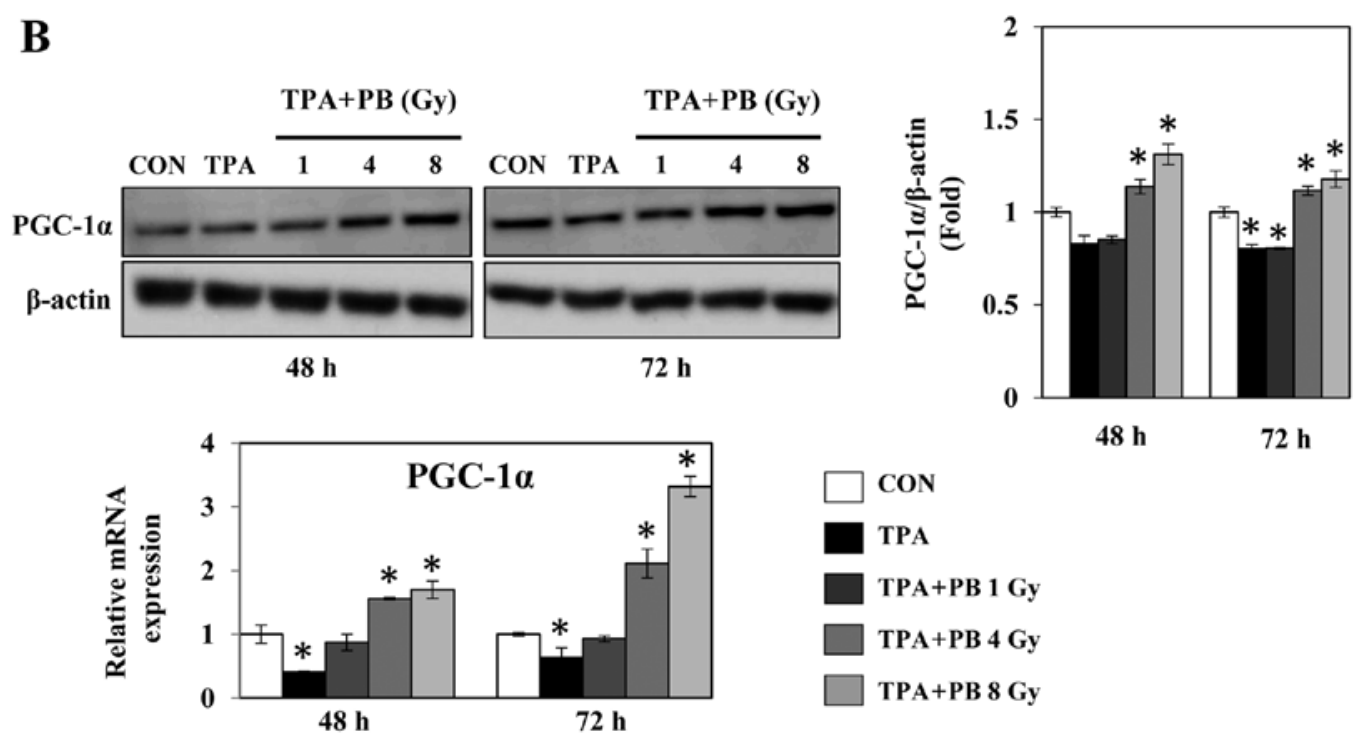

Figure 1. Effects of proton beam irradiation on mitochondrial biogenesis and PGC-1 $\alpha$ expression in 12- $O$-tetradecanoylphorbol-13-acetate (TPA)-induced aggressive HT-29 cells. After irradiation at the indicated doses, mitochondrial biogenesis was evaluated using quantitative reverse transcription polymerase chain reaction (RT-qPCR) to determine the ratio of the relative amount of mitochondrial DNA (COX I) to that of nuclear DNA (p0) at the indicated times (A). The expression of PGC-1 $\alpha$ was determined by western blotting and RT-qPCR analysis at the indicated times (B). The western blotting results for PGC-1 $\alpha$ were quantified using ImageJ software. Each value represents the mean $\pm \mathrm{SEM} .{ }^{*} \mathrm{P}<0.05,{ }^{* *} \mathrm{P}<0.01$ vs. the CON group.

overnight at $4^{\circ} \mathrm{C}$. After washing with Tris-buffered saline (TBS) containing $0.1 \%$ Tween-20 for $30 \mathrm{~min}$, the membranes were incubated with the anti-mouse IgG, or anti-rabbit IgG, HRP-conjugated secondary antibodies $(1: 3,000)$ for $1 \mathrm{~h}$ at $25^{\circ} \mathrm{C}$. After washing with TBS containing $0.1 \%$ Tween-20 for $30 \mathrm{~min}$, the western blotting images were obtained with an ECL Plus Western blotting substrate (Pierce Biotechnology), using a Lumino image analyzer (LAS 4000 mini; Fujifilm, Tokyo, Japan), and quantified with ImageJ software (ver. 1.51j; National Institutes of Health, Bethesda, MD, USA).

Statistical analysis. All data are shown as the mean \pm SEM. The data were analyzed by the one-way analysis of variance (ANOVA). Differences between mean values were assessed using the Dunnett's multiple comparisons test. Statistical significance was defined as $\mathrm{P}<0.05$.

\section{Results}

Proton beam irradiation stimulates mitochondrial biogenesis in TPA-induced aggressive HT-29 cells. To determine whether proton beam irradiation regulates mitochondrial biogenesis, we analyzed the gene expression ratio of COX I and RPLP0, representing mtDNA and nDNA, respectively, in TPA-induced aggressive HT-29 cells after irradiation with a proton beam at 1,4 or 8 Gy. Proton beam irradiation increased mitochondrial biogenesis in a dose- and timedependent manner (Fig. 1A). At the same time, proton beam irradiation increased the expression of PGC-1 $\alpha$, a key transcription factor for mitochondrial biogenesis, in a dose-dependent manner (Fig. 1B). To determine whether proton beam irradiation-induced mitochondrial biogenesis is involved in mitochondrial function, we next stained mitochondria in TPA-induced aggressive HT-29 cells using MitoTracker Red CMXRos, a mitochondrial membrane potential $(\Delta \Psi \mathrm{m})$-sensitive fluorochrome that accumulates membrane potential-dependently in live cells. Proton beam irradiation increased mitochondrial staining compared with staining in the group treated with TPA alone (Fig. 2). Therefore, these results indicate that proton beam irradiation-induced mitochondrial biogenesis is associated with the inhibitory effects of proton beam irradiation on metastatic potential in TPA-induced aggressive HT-29 cells.

Proton beam irradiation stimulates mitochondrial biogenesis by AMPK signaling pathways in TPA-induced aggressive HT-29 cells. To further study the mechanism by which proton beam irradiation affects mitochondrial biogenesis and function, we next investigated the phosphorylation of AMPK and SIRT1, which are upstream molecules associated with PGC-1 $\alpha$ 
Table I. Primers for quantitative RT-PCR analysis.

\begin{tabular}{lll}
\hline & \multicolumn{1}{c}{ Forward } & \multicolumn{1}{c}{ Reverse } \\
\hline PGC-1 $\alpha$ & GTTGCCTGCATGAGTGTGTG & TCACTGCACCACTTGAGTCC \\
mtTFA & ATGCTTATAGGGCGGAGTGG & CTTCAGCTTTCCTGCGGTG \\
ERF1 & AGAACTCTCCCTGCTGGACT & AGCACACTTACACGACGACT \\
TOMM40 & GCGATGTCCTTTTTGTCCT & TCCGAGGAACCCTTTGACT \\
TIMM44 & CTCTGACCTCTCCCCTAGCAG & CGATTGTGCTGAGGGCTACT \\
CPT1 $\alpha$ & TGCTACGGAAGAAGCTTGGG & CTTCTTCACGGACTCCACCC \\
CytC & TTCAGTTCACGGTCACTCCG & TGACCACGTTCTTCGTCTGG \\
ATP5B & CAGTGCCACACCGTTGAAAA & TGCATCGGTTATTCACACTCC \\
RPLP0 & TGCCCCTGCTACTACGTTTG & TGGCTGAGACAAGAAACGCT \\
COX1 & CCTTCTCCTTTGGGCTGGTCATCCA & CAGACACTGGCAACATTGCGGACAC \\
$\beta$-actin & TCACCCACACTGTGCCCATCTACGA & CAGCGGAACCGCTCATTGCCAATGG \\
\hline
\end{tabular}

PGC-1 $\alpha$, PPAR gamma coactivator-1 $\alpha$; mtTFA, mitochondrial transcription factor A; NRF1, nuclear respiratory factor-1; ERR $\alpha$, estrogen related receptor alpha; TOMM40, translocase of outer mitochondrial membrane 40; TIMM44, translocase of inner mitochondrial membrane 44; CPT1 $\alpha$, carnitine palmitoyltransferase $1 \alpha$; CytC, cytochrome $c$; ATP5B, ATP synthase subunit $\beta$, mitochondrial; RPLP0, 60S acidic ribosomal protein P0; COX1, cytochrome $c$ oxidase subunit I.
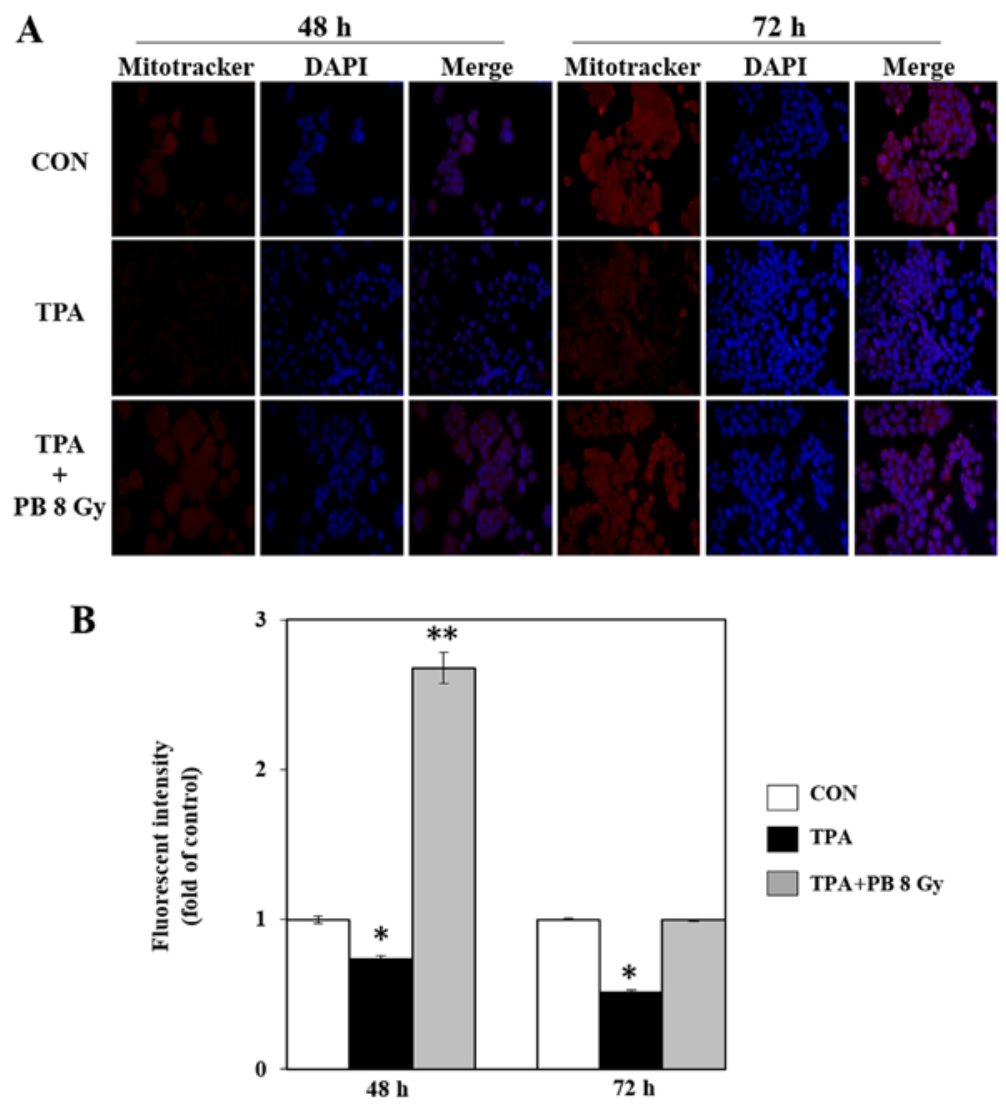

Figure 2. Effects of proton beam irradiation on mitochondrial membrane potential in 12 - $O$-tetradecanoylphorbol-13-acetate (TPA)-induced HT-29 cells For image acquisition, cells were incubated with RPMI-1640 medium containing $20 \mathrm{nM}$ MitoTracker Red CMXRos for 30 min at the indicated times. (A) Representative MitoTracker Red CMXRos and DAPI images are shown at x400 magnification. (B) The fluorescent signal intensities of MitoTracker Red CMXRos were quantified using a plate reader (average values, $\mathrm{n}=10$ ). Each value represents the mean $\pm \mathrm{SEM}$. ${ }^{*} \mathrm{P}<0.05,{ }^{* * *} \mathrm{P}<0.01$ vs. the CON group.

and regulators of mitochondrial biogenesis. As shown in Fig. 3, TPA treatment decreased AMPK phosphorylation, whereas proton beam irradiation increased AMPK phosphorylation in a dose- and time-dependent manner. However, proton beam irradiation did not affect SIRT1 phosphorylation. Therefore, these results indicate that the proton beam irradiation-induced increase in mitochondrial biogenesis might be regulated by the AMPK signaling pathways. 
A
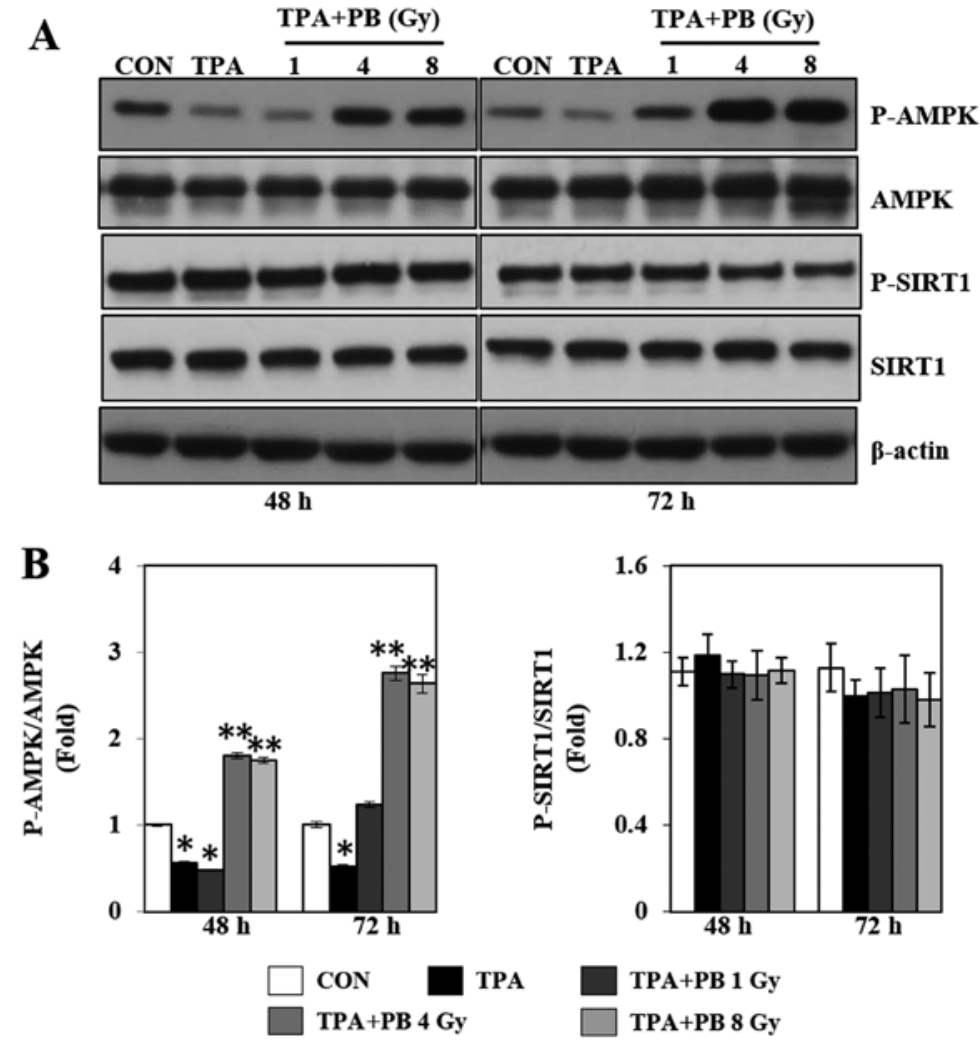

Figure 3. Effects of proton beam irradiation on AMPK and SIRT1 activity in 12- $O$-tetradecanoylphorbol-13-acetate (TPA)-induced aggressive HT-29 cells. (A) After irradiation at the indicated doses, western blot analysis was performed with anti-phospho AMPK, anti-AMPK, anti-phospho SIRT1 and anti-SIRT1 antibodies at the indicated times. (B) Quantitative analysis of western blot results was performed using ImageJ software. Each value represents the mean \pm SEM. ${ }^{*} \mathrm{P}<0.05,{ }^{* *} \mathrm{P}<0.01$ vs. the $\mathrm{CON}$ group.
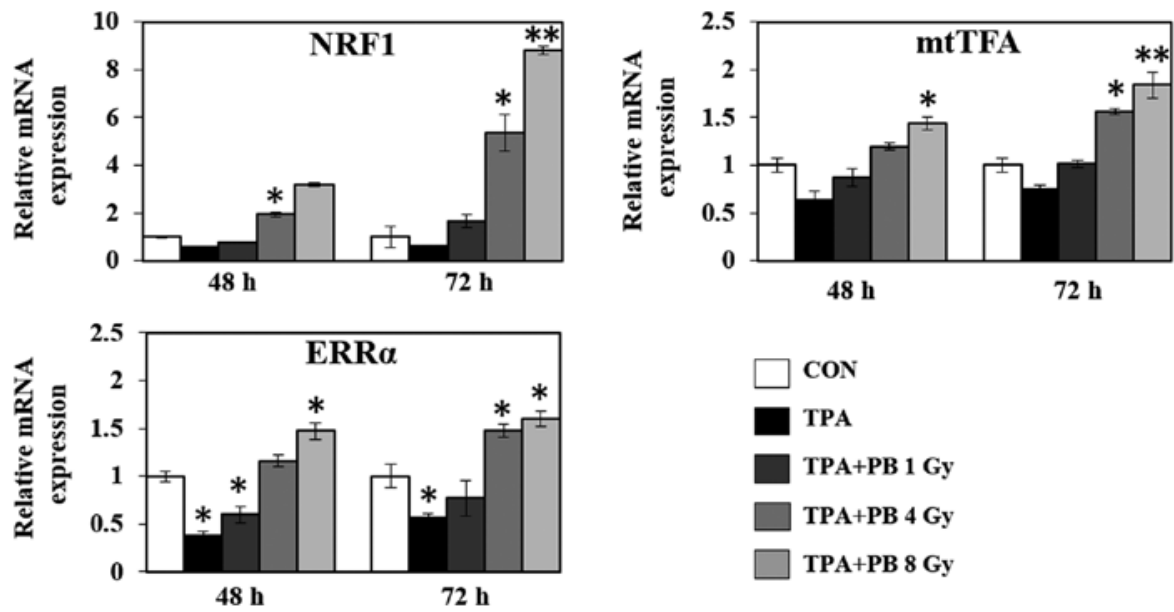

Figure 4. Effect of proton beam irradiation on gene expression of mitochondrial key transcription factors in 12-O-tetradecanoylphorbol-13-acetate (TPA)-induced aggressive HT-29 cells. After irradiation at the indicated doses, the expression levels of NRF1, mtTFA and ERR $\alpha$ were determined by quantitative reverse transcription polymerase chain reaction (RT-qPCR) at the indicated times. Each value represents the mean $\pm \mathrm{SEM}$. " $\mathrm{P}<0.05$, ${ }^{* *} \mathrm{P}<0.01 \mathrm{vs}$. the CON group.

Proton beam irradiation stimulates the expression of mitochondrial transcription factors and mitochondria functional genes in TPA-induced aggressive HT-29 cells. To further investigate the underlying regulatory mechanism associated with mitochondrial biogenesis, we investigated the mRNA expression of various genes involved in mitochondrial transcription, mitochondrial dynamics, the electron transport chain, ATP synthesis and fatty acid oxidation. As shown in Fig. 4, proton beam irradiation increased the gene expression of the transcriptional co-activators NRF1, mtTFA and ERR $\alpha$ in a dose- and time-dependent manner. At the same time, proton beam irradiation increased the gene expression of dynamin-related protein 1 (DRP1; a GTPase that regulates mitochondrial fission), optic atrophy 1 (OPA1; a dynaminrelated GTPase, which participates in mitochondrial fusion and mitochondrial cristae remodeling), translocase of outer 

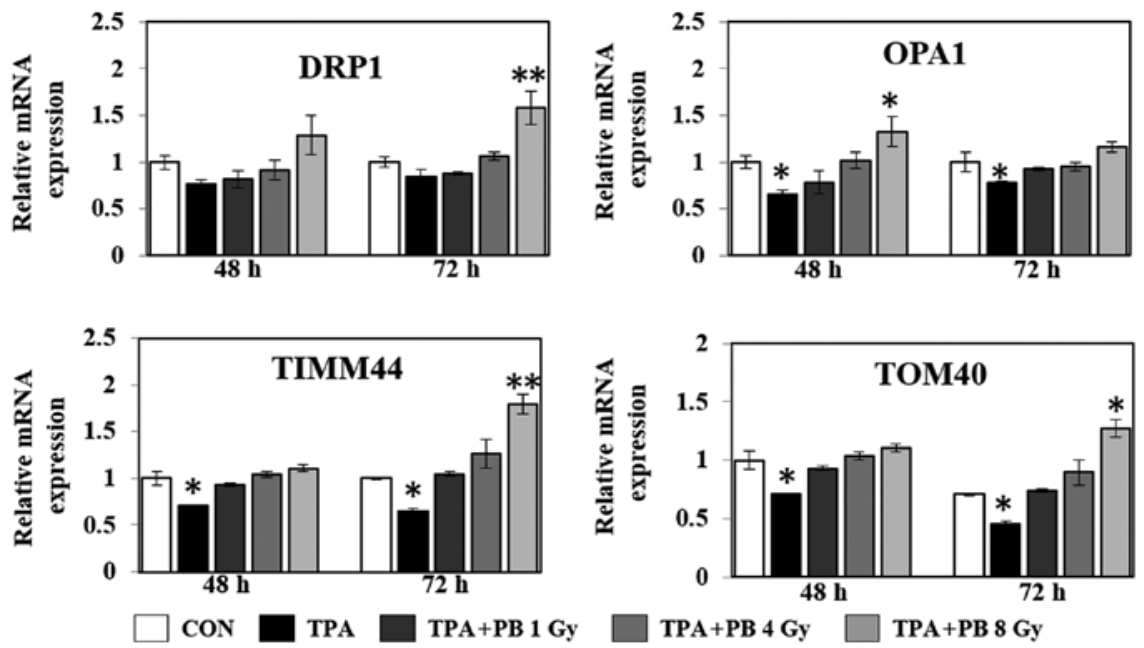

Figure 5. Effect of proton beam irradiation on gene expression related to mitochondrial dynamics in 12- $O$-tetradecanoylphorbol-13-acetate (TPA)-induced aggressive HT-29 cells. After irradiation at the indicated doses, the expression levels of DRP1, OPA1, TIMM44 and TOM40 were determined by quantitative reverse transcription polymerase chain reaction (RT-qPCR) at the indicated times. Each value represents the mean $\pm \mathrm{SEM}$. "P<0.05, ${ }^{* *} \mathrm{P}<0.01$ vs. the CON group.
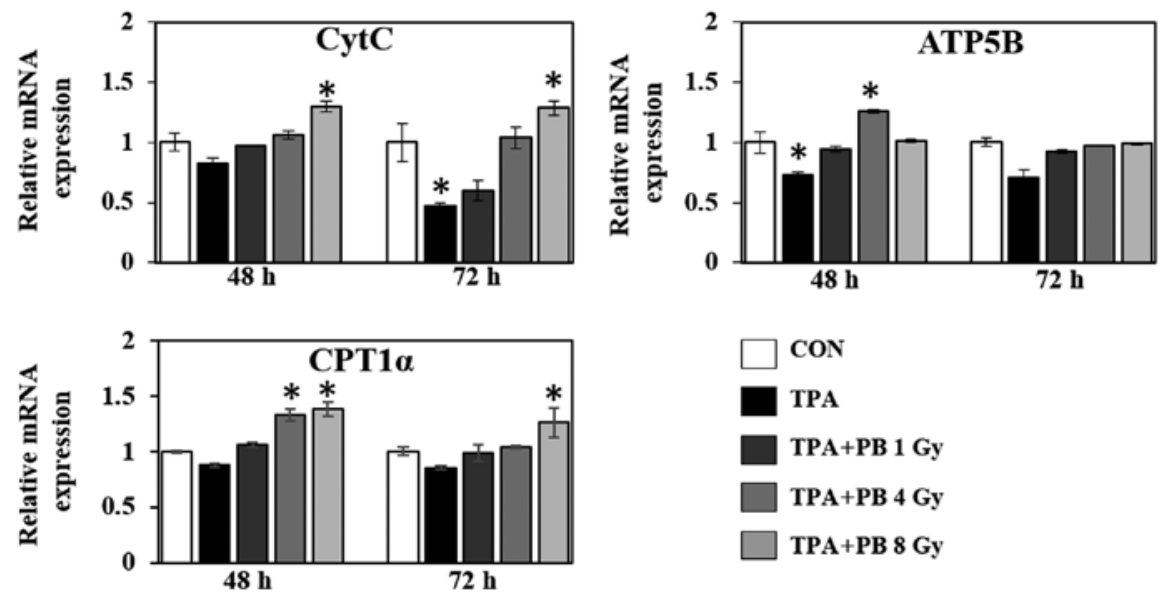

Figure 6. Effect of proton beam irradiation on gene expression related to mitochondrial function in 12-O-tetradecanoylphorbol-13-acetate (TPA)-induced aggressive HT-29 cells. After irradiation at the indicated doses, the expression levels of CytC, ATP5B and CTP-1 $\alpha$ were determined by quantitative reverse transcription polymerase chain reaction (RT-qPCR) at the indicated times. Each value represents the mean $\pm \mathrm{SEM}$. ${ }^{*} \mathrm{P}<0.05$, ${ }^{* *} \mathrm{P}<0.01$ vs. the CON group.

mitochondrial membrane 40 (TOMM40), and translocase of inner mitochondrial membrane 44 (TIMM44), which play a role in mitochondrial protein import (Fig. 5). Moreover, in the context of mitochondrial function, proton beam irradiation increased the gene expression of cytochrome $c(\mathrm{CytC})$, an essential component of the electron transport chain, ATP synthase subunit $\beta$ (ATP5B), a subunit of mitochondrial ATP synthase, and carnitine palmitoyltransferase 1- $\alpha$ (CPT1- $\alpha$ ), a mitochondrial enzyme responsible for the $\beta$-oxidation of long-chain fatty acids, in a dose- and time-dependent manner (Fig. 6). Therefore, these results indicate that proton beam irradiation is an effective stimulator of mitochondrial biogenesis and function in TPA-induced aggressive HT-29 cells.

\section{Discussion}

Proton beam therapy (PBT) is a new form of radiotherapy used to treat cancer. Compared with other radiotherapies such as gamma and X-ray, PBT has better dose distribution owing to its unique absorption spectrum in tissues, known as the Bragg's peak, which allows the deposition of devastating energy at the tumor while limiting the damage to normal tissues (21). However, the biological effects and detailed mechanism of proton beam irradiation on cancer metabolism are still unclear, and further studies are needed to determine the biological role of proton beam irradiation for efficient application. In the present study, we proposed that proton beam irradiation suppresses colon cancer metastasis by stimulating mitochondrial biogenesis and inducing the expression of mitochondrial transcription factors, functional regulators, and dynamic-related molecules, and by upregulating AMPK activity in TPA-induced aggressive HT-29 cells.

PGC- $1 \alpha$ is the main regulator of mitochondrial biogenesis, and acts through interactions with transcription factors such as NRF1 and ERR $\alpha$ (4). It is sensitive to cellular energy status, which is influenced by cold, exercise and fasting and its activity is closely controlled by several post-translational modifications 
such as phosphorylation and acetylation. Both AMPK-mediated phosphorylation and SIRT1-mediated deacetylation activate PGC-1 $\alpha$ under energy deprivation conditions (21). NRF-1 regulates mtTFA expression, thereby mediating the increased expression of nuclear mitochondrial genes with increased mtDNA replication and expression $(22,23)$. ERR $\alpha$ acts as a co-transcription factor of PGC- $1 \alpha$, and regulates the expression of genes involved in fatty acid oxidation, the TCA cycle, mitochondrial biogenesis and dynamics and OXPHOS. ERR $\alpha$ also acts both directly on genes involved in mitochondrial structural components and enzymes (24). Recent studies have revealed that the PGC- $1 \alpha$-mediated transcriptional cascade suppresses invasion and migration in different types of cancer cells. Integrative metabolomics and transcriptomics using genetically engineered mouse models and xenografts have shown that PGC-1 $\alpha$ activates an ERR $\alpha$-dependent transcriptional network to induce a catabolic state and suppress metastasis in prostate cancer (25). In melanoma, PGC-1 $\alpha$ directly increases the transcription of DNA-binding protein inhibitor (ID2), which in turn binds to and inactivates transcription factor 4 (TCF4). TCF4 inactivation downregulates metastasis-related genes including integrins and integrinmediated signaling pathways that play a role in invasion and migration (26). In the present study, we also found that proton beam irradiation effectively increased mitochondrial biogenesis by upregulating PGC- $1 \alpha$ expression and its co-transcriptional factors such as NRF1 and ERR $\alpha$, as well as the mitochondrial transcription factor mtTFA. Therefore, our findings demonstrate that increased mitochondrial biogenesis, dynamics, and function by a PGC- $1 \alpha$-mediated transcriptional cascade might be a novel mechanism underlying the inhibitory effects of proton beam irradiation on metastatic potential in TPA-induced aggressive HT-29 cells.

AMPK is a hetero-trimeric protein complex composed of a catalytic subunit and two regulatory subunits; it plays an essential role in regulating metabolic and energy homeostasis (27). In particular, AMPK is well known as the main regulator for lipid, cholesterol and glucose metabolism in various tissues, such as the liver, muscles and adipose tissues. Owing to this physiological role, AMPK has been considered a key therapeutic target in patients with metabolic diseases such as obesity and diabetes (28). Moreover, therapeutic manipulation for targeting AMPK signal pathways using some diabetes drugs in cancer patients shows the association between AMPK and several tumor suppressors (29). Recent studies have indicated that AMPK not only inhibits cancer cell proliferation but also invasion. The siRNA-mediated knockdown and pharmacologic activation of AMPK by OSU-53, a novel allosteric AMPK activator, suppresses the epithelial-mesenchymal transition (EMT) by modulating Akt-MDM2-Foxo3 signaling in breast and prostate cancer cells (30). Treatment with metformin, an AMPK activator, inhibits EMT in an AMPK/ p53-dependent manner in metastatic melanoma cells (31) and inhibits both angiogenesis and metastatic spread in ovarian cancer (32). Similarly, Kim et al (33) reported that AMPK activation by berberine, another AMPK activator, suppresses human melanoma cell migration by decreasing Erk activity and cytochrome $c$ oxidase subunit II expression. Notably, our findings showed that proton beam irradiation increased TPA-inhibited AMPK phosphorylation, which supports the increased expression of PGC1- $\alpha$ and its co-transcription factors including NRF1 and ERR $\alpha$, as well as target genes including CytC, ATP5B and CPT-1 $\alpha$, which are associated with OXPHOS components and mitochondrial functional enzymes. Collectively, AMPK activation by proton beam irradiation is decisive for mitochondrial biogenesis, and may exert a tumor suppressor function and enhance mitochondrial function. However, the specific role of AMPK activation by proton beam irradiation on metastasis and mitochondrial function requires further study.

Mitochondria are highly dynamic organelles that are continuously undergoing fusion and fission; they are necessary for maintaining cellular physiological functions such as survival, death and metabolic homeostasis (34). Although mitochondrial dysfunction has been associated with tumorigenesis, the roles of mitochondrial dynamics in metastasis are poorly understood. Altered mitochondrial dynamics have been associated with changed mitochondrial physiology and cell dysfunction (35), which are involved in many human diseases such as cancer, neurodegenerative diseases including Alzheimer's, Huntington's and Parkinson's diseases, and cardiometabolic diseases including diabetes and cardiomyopathy (36). A recent study showed that human lung cancer cells have excess mitochondrial fission and impaired mitochondrial fusion due to imbalanced DRP1/MFN expression (37). Our findings suggest that proton beam irradiation effectively regulates dysfunctional mitochondrial dynamics in metastatic cancer by increasing the expression levels of DRP1 and OPA1.

Over $99 \%$ of proteins involved in mitochondrial biogenesis are imported from the cytoplasm. This mitochondrial protein import process occurs through channel protein complexes such as the translocase of the outer membrane (TOM) and the translocases of the inner membrane (TIM) (38). Mitochondrial membrane channels such as ion and protein channels are considered promising therapeutic targets for cancer transformation owing to their correlation in metabolic and apoptotic functions (39). In particular, many subunits of the mitochondrial protein import complexes are dysregulated in cancer cell mitochondria (40). The mitochondrial protein import system subunits TIMM44 and TOM40 play an important role in mitochondrial functions such as the import of OXPHOS-related proteins and mitochondrial redox balance (41). Our findings show that proton beam irradiation might be an effective regulator of TIMM44 and TOM40 expression.

In conclusion, our findings show that proton beam irradiation increases mitochondrial biogenesis by modulating the expression of the main mitochondrial transcription factors, functional regulators and dynamic-related molecules by inducing AMPK activity in TPA-induced aggressive HT-29 cells. We propose that targeting mitochondrial biogenesis may be a novel inhibitory mechanism for metastatic cancer metabolism.

\section{Acknowledgements}

The present study was supported by the National Nuclear R\&D Program through the National Research Foundation (NRF) of Korea funded by the Ministry of Science, ICT and Future Planning (NRF- 2016M2B2A4910909). 


\section{References}

1. Favoriti P, Carbone G, Greco M, Pirozzi F, Pirozzi RE and Corcione F: Worldwide burden of colorectal cancer: A review. Updates Surg 68: 7-11, 2016.

2. Steeg PS: Targeting metastasis. Nat Rev Cancer 16: 201-218, 2016.

3. Suliman HB and Piantadosi CA: Mitochondrial quality control as a therapeutic target. Pharmacol Rev 68: 20-48, 2016.

4. Scarpulla RC, Vega RB and Kelly DP: Transcriptional integration of mitochondrial biogenesis. Trends Endocrinol Metab 23: 459-466, 2012

5. Biswas G, Guha M and Avadhani NG: Mitochondria-to-nucleus stress signaling in mammalian cells: Nature of nuclear gene targets, transcription regulation, and induced resistance to apoptosis. Gene 354: 132-139, 2005.

6. van Waveren C, Sun Y, Cheung HS and Moraes CT: Oxidative phosphorylation dysfunction modulates expression of extracellular matrix--remodeling genes and invasion. Carcinogenesis 27: 409-418, 2006

7. Wang $X$ and Moraes CT: Increases in mitochondrial biogenesis impair carcinogenesis at multiple levels. Mol Oncol 5: 399-409, 2011.

8. Liu W, Beck BH, Vaidya KS, Nash KT, Feeley KP, Ballinger SW, Pounds KM, Denning WL, Diers AR, Landar A, et al: Metastasis suppressor KISS1 seems to reverse the Warburg effect by enhancing mitochondrial biogenesis. Cancer Res 74: 954-963, 2014.

9. Mohan R and Grosshans D: Proton therapy - Present and future. Adv Drug Deliv Rev 109: 26-44, 2016.

10. Fukuda K, Okumura T, Abei M, Fukumitsu N, Ishige K, Mizumoto M, Hasegawa N, Numajiri $\mathrm{H}$, Ohnishi K, Ishikawa $\mathrm{H}$, et al: Long-term outcomes of proton beam therapy in patients with previously untreated hepatocellular carcinoma. Cancer Sci 108: 497-503, 2016

11. Hatayama $Y$, Nakamura $T$, Suzuki M, Azami $Y$, Ono $T$, Yabuuchi T, Hayashi Y, Kimura K, Hirose K, Wada $\mathrm{H}$, et al: Clinical outcomes and prognostic factors of high-dose proton beam therapy for peripheral stage I non-Small-cell lung cancer. Clin Lung Cancer 17: 427-432, 2016.

12. Yamoah $\mathrm{K}$ and Johnstone PA: Proton beam therapy: Clinical utility and current status in prostate cancer. Onco Targets Ther 9: 5721-5727, 2016

13. Romesser PB, Cahlon O, Scher E, Zhou Y, Berry SL, Rybkin A, Sine KM, Tang S, Sherman EJ, Wong R, et al: Proton beam radiation therapy results in significantly reduced toxicity compared with intensity-modulated radiation therapy for head and neck tumors that require ipsilateral radiation. Radiother Oncol 118: 286-292, 2016.

14. Ogata T, Teshima T, Kagawa K, Hishikawa Y, Takahashi Y, Kawaguchi A, Suzumoto Y, Nojima K, Furusawa Y and Matsuura N: Particle irradiation suppresses metastatic potential of cancer cells. Cancer Res 65: 113-120, 2005.

15. Narang H, Kumar A, Bhat N, Pandey BN and Ghosh A: Effect of proton and gamma irradiation on human lung carcinoma cells: Gene expression, cell cycle, cell death, epithelial-mesenchymal transition and cancer-stem cell trait as biological end points. Mutat Res 780: 35-46, 2015.

16. Nam KS, Kim MK and Shon YH: Cancer chemopreventive enzymes of human colorectal adenocarcinoma cells irradiated with proton beams. J Korean Phys Soc 52: 945-948, 2008.

17. Nam KS and Shon YH: Suppression of metastatic potential in human colorectal adenocarcinoma cells irradiated with proton beams. J Korean Phys Soc 59: 709-712, 2011.

18. Ha BG, Park JE, Cho HJ, Lim YB and Shon YH: Inhibitory effects of proton beam irradiation on integrin expression and signaling pathway in human colon carcinoma HT29 cells. Int J Oncol 46: 2621-2628, 2015.

19. Kim B, Bae H, Lee H, Lee S, Park JC, Kim KR and Kim SJ: Proton beams inhibit proliferation of breast cancer cells by altering DNA methylation status. J Cancer 7: 344-352, 2016.

20. Ha BG, Park JE, Cho HJ and Shon YH: Stimulatory effects of balanced deep sea water on mitochondrial biogenesis and function. PLoS One 10: e0129972, 2015.
21. MacDonald SM, DeLaney TF and Loeffler JS: Proton beam radiation therapy. Cancer Invest 24: 199-208, 2006.

22. Wenz T: Regulation of mitochondrial biogenesis and PGC-1 $\alpha$ under cellular stress. Mitochondrion 13: 134-142, 2013.

23. Zhang Y and Xiang Y: Molecular and cellular basis for the unique functioning of $\mathrm{Nrf1}$, an indispensable transcription factor for maintaining cell homoeostasis and organ integrity. Biochem J 473: 961-1000, 2016.

24. Villena JA and Kralli A: ERRalpha: A metabolic function for the oldest orphan. Trends Endocrinol Metab 19: 269-276, 2008.

25. Torrano V, Valcarcel-Jimenez L, Cortazar AR, Liu X, Urosevic J, Castillo-Martin M, Fernández-Ruiz S, Morciano G, CaroMaldonado A, Guiu M, et al: The metabolic co-regulator PGC1a suppresses prostate cancer metastasis. Nat Cell Biol 18: 645-656, 2016.

26. Luo C, Lim JH, Lee Y, Granter SR, Thomas A, Vazquez F, Widlund HR and Puigserver P: A PGC1 $\alpha$-mediated transcriptional axis suppresses melanoma metastasis. Nature 537: 422-426, 2016

27. Hardie DG, Schaffer BE and Brunet A: AMPK: An energysensing pathway with multiple inputs and outputs. Trends Cell Biol 26: 190-201, 2016.

28. Gasparrini M, Giampieri F, Alvarez Suarez JM, Mazzoni L, Y Forbes Hernandez T, Quiles JL, Bullon P and Battino M: AMPK as a new attractive therapeutic target for disease prevention: The role of dietary compounds AMPK and disease prevention. Curr Drug Targets 17: 865-889, 2016.

29. Shackelford DB and Shaw RJ: The LKB1-AMPK pathway: Metabolism and growth control in tumour suppression. Nat Rev Cancer 9: 563-575, 2009.

30. Chou CC, Lee KH, Lai IL, Wang D, Mo X, Kulp SK, Shapiro CL and Chen CS: AMPK reverses the mesenchymal phenotype of cancer cells by targeting the Akt-MDM2-Foxo3a signaling axis. Cancer Res 74: 4783-4795, 2014.

31. Cerezo M, Tichet M, Abbe P, Ohanna M, Lehraiki A, Rouaud F, Allegra M, Giacchero D, Bahadoran P, Bertolotto C, et al: Metformin blocks melanoma invasion and metastasis development in AMPK/p53-dependent manner. Mol Cancer Ther 12: $1605-1615,2013$

32. Rattan R, Graham RP, Maguire JL, Giri S and Shridhar V: Metformin suppresses ovarian cancer growth and metastasis with enhancement of cisplatin cytotoxicity in vivo. Neoplasia 13: 483-491, 2011.

33. Kim HS, Kim MJ, Kim EJ, Yang Y, Lee MS and Lim JS: Berberine-induced AMPK activation inhibits the metastatic potential of melanoma cells via reduction of ERK activity and COX-2 protein expression. Biochem Pharmacol 83: 385-394, 2012.

34. Westermann B: Mitochondrial fusion and fission in cell life and death. Nat Rev Mol Cell Biol 11: 872-884, 2010.

35. Detmer SA and Chan DC: Functions and dysfunctions of mitochondrial dynamics. Nat Rev Mol Cell Biol 8: 870-879, 2007.

36. Mishra P: Interfaces between mitochondrial dynamics and disease. Cell Calcium 60: 190-198, 2016.

37. Zhao J, Zhang J, Yu M, Xie Y, Huang Y, Wolff DW, Abel PW and $\mathrm{Tu}$ Y: Mitochondrial dynamics regulates migration and invasion of breast cancer cells. Oncogene 32: 4814-4824, 2013.

38. Chacinska A, Koehler CM, Milenkovic D, Lithgow $\mathrm{T}$ and Pfanner N: Importing mitochondrial proteins: Machineries and mechanisms. Cell 138: 628-644, 2009.

39. Madamba SM, Damri KN, Dejean LM and Peixoto PM: Mitochondrial ion channels in cancer transformation. Front Oncol 5: 120, 2015.

40. Sotgia F, Whitaker-Menezes D, Martinez-Outschoorn UE, Salem AF, Tsirigos A, Lamb R, Sneddon S, Hulit J, Howell A and Lisanti MP: Mitochondria 'fuel' breast cancer metabolism: Fifteen markers of mitochondrial biogenesis label epithelial cancer cells, but are excluded from adjacent stromal cells. Cell Cycle 11: 4390-4401, 2012.

41. Baker MJ, Frazier AE, Gulbis JM and Ryan MT: Mitochondrial protein-import machinery: Correlating structure with function. Trends Cell Biol 17: 456-464, 2007. 